\title{
La industria de la leche en Nueva Zelanda
}

\section{Introducción}

$\mathrm{E}$ $n$ este artículo se presenta un panorama general de la industria lechera de Nueva Zelanda, entendiendo como industria a toda la cadena de producción y comercialización de la leche. Se analizan algunas de las razones que han permitido que el sistema de producción de leche en Nueva Zelanda sea uno de los más competitivos dentro de los países desarrollados, así como la importancia relativa de este país en el mercado internacional de lácteos y los cambios estructurales que se han dado en los últimos años en la industria. Finalmente, se abordan los elevados subsidios que se aplican sobre todo en países desarrollados, y el problema que representan para los productores de leche, principalmente en países pobres.

\section{Características de la industria}

La industria lechera de Nueva Zelanda es una de las más importantes en el mundo debido a su competitividad y a su importante presencia en los mercados internacionales. Una característica importante la constituye su sistema de producción basado en el pastoreo, el cual es poco común a escala internacional. Actualmente se calcula que aproximadamente sólo 10 por

* Profesor investigador del Departamento de Estudios del Pacífico de la Universidad de Guadalajara. ciento del ganado lechero en el mundo tiene como principal componente de alimentación el pasto; esta forma de explotación se encuentra en Nueva Zelanda, Australia y algunas zonas de Sudáfrica y Sudamérica. ${ }^{1}$ A diferencia de los sistemas de producción estabulados, donde el ganado permanece gran parte del tiempo en los establos y su dieta se compone de forraje y granos, las condiciones naturales de Nueva Zelanda, que incluyen suelos con una abundante capa de materia orgánica, distribución excelente de lluvias y un invierno benigno, permiten evitar la estabulación invernal y contar con pastos durante todo el año sin necesidad de riego. ${ }^{2}$ Esta forma de explotación necesariamente requiere de un espacio amplio para el ganado, y es así como está organizado. Actualmente, en Nueva Zelanda existen aproximadamente 13,500 granjas lecheras y, en promedio, cada una cuenta con 250 bovinos y una extensión de 100 hectáreas, es decir, 2.5 cabezas de ganado por hectárea.

Las características naturales mencionadas, además del desarrollo de innovaciones tecnológicas, han hecho posible que la producción de leche en Nueva Zelanda se realice con los costos de producción más bajos registrados en países desarrollados; sin embargo, es precisamente el sistema de producción basado en el pastoreo con bajo uso de granos, lo que también provoca rendimientos inferiores en la productividad, hasta en 50 por ciento respecto a las 
Cuadro 1

Promedio anual de producción de leche por vaca (litros)

\begin{tabular}{llllll}
\hline País & 2000 & 2001 & 2002 & 2003 & 2004 \\
\hline Nueva Zelanda & 3,666 & 3,689 & 3,700 & 3,736 & 3,667 \\
Australia & 5,151 & 4,996 & 5,473 & 5,186 & 5,111 \\
Unión Europea* & 6,007 & 5,999 & 6,078 & 6,284 & n.d \\
Estados Unidos & 8,254 & 8,235 & 8,438 & 8,504 & 8,636 \\
México & 1,369 & 1,393 & 1,245 & 1,375 & 1,437 \\
Mundo & 2,153 & 2,165 & 2,173 & 2,178 & 2,172 \\
\hline
\end{tabular}

n.d. = no disponible

* Incluye 15 países (Austria, Bélgica, Dinamarca, Finlandia, Francia, Alemania, Grecia, Irlanda Italia, Luxemburgo, Holanda, Portugal, España, Inglaterra y Suecia).

Fuente: www.fao.org

granjas con manejo estabulado y uso de concentrados. ${ }^{3}$

Como se puede observar en el cuadro 1, mientras en Nueva Zelanda cada bovino produjo poco más de 3,500 litros de leche en promedio anual durante los últimos cuatro años, en Estados Unidos la producción fue de más del doble, en Europa cercana al doble, y en Australia alrededor de 30 por ciento más de la que se registró en Nueva Zelanda. Aunque los sistemas de producción en Nueva Zelanda y Australia son parecidos, uno de los aspectos importantes que los distinguen es que en Nueva Zelanda su sistema se basa en mayor medida en el pastoreo, con mucho menos uso de suplementos alimenticios que en el sistema de producción australiano. ${ }^{4}$

Por lo mencionado, Nueva Zelanda y Australia constituyen dos casos excepcionales (en el grupo de países desarrollados) por sus sistemas de producción de ganado lechero. Su competitividad se ha logrado en mayor medida debido a las ventajas comparativas y al menor grado de intervención de las políticas gubernamentales. En los casos de los principales países productores de leche, como los de la Unión Europea y Estados Unidos, que también son competitivos en la producción de leche, su competitividad se ha logrado principalmente por la intervención de las políticas gubernamentales. Por lo tanto, podemos decir que en estos países la competitividad se percibe como el resultado combinado de los efectos de las distorsiones de mercado (provocadas tanto por políticas como por la competencia imperfecta) y de las ventajas comparativas. ${ }^{5}$

\section{Participación de Nueva Zelanda en el mercado internacional}

La mayoría de los países consumen casi el total de la leche que producen, y sólo una parte marginal se destina al comercio internacional. Actualmente poco menos de 10 por ciento de la producción mundial es la que se comercia internacionalmente, excluyendo lo que se comercia entre los países de la Unión Europea. Así, en el año 2003 el comercio internacional de lácteos en el mundo, incluyendo el comercio entre países de la Unión Europea, fue de 77,842 millones de litros, es decir, 15 por ciento de la producción mundial; en cambio, si descontáramos el comercio realizado entre estos países, el comercio mundial de lácteos 
Cuadro 2

Comercio internacional de lácteos, exportaciones

\begin{tabular}{lrrrrrrrr}
\hline & \multicolumn{1}{c}{ El equivalente a millones de litros } & \multicolumn{5}{c}{ Millones de dólares US } \\
\hline & \multicolumn{1}{c}{ 2002 } & \multicolumn{1}{c}{$\%$} & \multicolumn{1}{c}{ 2003 } & \multicolumn{1}{c}{$\%$} & \multicolumn{1}{c}{2002} & $\%$ & 2003 & $\%$ \\
\hline Nueva Zelanda & 11,034 & 14.97 & 11,352 & 14.58 & 2,592 & 9.70 & 2,932 & 9.00 \\
Australia & 6,118 & 8.30 & 4,540 & 5.83 & 1,578 & 5.90 & 1,348 & 4.14 \\
Unión Europea* & 40,846 & 55.41 & 45,911 & 58.98 & 18,042 & 67.50 & 23,333 & 71.60 \\
Estados Unidos & 2,616 & 3.55 & 2,826 & 3.63 & 540 & 2.02 & 610 & 1.87 \\
México & 105 & 0.14 & 109 & 0.14 & 44 & 0.16 & 47 & 0.14 \\
Mundo & 73,712 & 100.00 & 77,842 & 100.00 & 26,730 & 100.00 & 32,590 & 100.00 \\
* Con comercio intraeuropa. & & & & & & & & \\
$\quad$ Fuente: http://www.fao.org & & & & & & & &
\end{tabular}

Comercio internacional de lácteos, exportaciones

\begin{tabular}{|c|c|c|c|c|c|c|c|c|}
\hline & \multicolumn{4}{|c|}{ El equivalente a millones de litros } & \multicolumn{4}{|c|}{ Millones de dólares US } \\
\hline & 2002 & $\%$ & 2003 & $\%$ & 2002 & $\%$ & 2003 & $\%$ \\
\hline Nueva Zelanda & 11,034 & 25.11 & 11,352 & 25.62 & 2,592 & 19.88 & 2,932 & 20.27 \\
\hline Australia & 6,118 & 13.92 & 4,540 & 10.25 & 1,578 & 12.10 & 1,348 & 9.32 \\
\hline Unión Europea* & 11,105 & 25.27 & 12,372 & 27.93 & 4,349 & 33.36 & 5,211 & 36.02 \\
\hline Estados Unidos & 2,616 & 5.95 & 2,826 & 6.38 & 540 & 4.14 & 610 & 4.22 \\
\hline México & 105 & 0.24 & 109 & 0.25 & 44 & 0.34 & 47 & 0.32 \\
\hline Mundo & 43,941 & 100.00 & 44,303 & 100.00 & 13,037 & 100.00 & 14,468 & 100.00 \\
\hline
\end{tabular}

* Sin comercio intraeuropa.

Fuente: http://www.fao.org

sería de 44,303 millones de litros de leche, u 8.5 por ciento del total mundial.

Nueva Zelanda es el único país en el mundo que exporta cerca de 80 por ciento de su producción interna de leche. Esta diferencia con respecto a otras naciones se debe a que se ha desarrollado una industria lechera muy competitiva frente a un mercado interno que es relativamente pequeño, hecho que lo convierte en el mayor exportador de lácteos del mundo. Si se analizan las exportaciones mundiales de leche, incluido el comercio que se efectúa entre países de la Unión Europea, ${ }^{6}$ a esta última región correspondería 58 por ciento del comercio total y a Nueva Zelanda 14 por ciento (2003); más aún, si se excluye el comercio de lácteos al interior de la Unión Europea y sólo se contabilizan sus exportaciones fuera de la región, la participación de esta última en el comercio mundial disminuiría a 27 por ciento y la de Nueva Zelanda se incrementaría a 25 por ciento, es decir Nueva Zelanda por sí sola estaría contribuyendo con un cuarto del total de exportaciones lácteas en el mundo.

\section{Cuadro 3}

Producción mundial de leche (miles de toneladas métricas)

\begin{tabular}{lrrrr}
\hline & 2000 & \multicolumn{1}{c}{2001} & \multicolumn{1}{c}{2002} & \multicolumn{1}{c}{2003} \\
\hline Nueva & 12,235 & 13,119 & 13,865 & 14,354 \\
Zelanda & & & & \\
Australia & 11,183 & 10,872 & 11,620 & 10,642 \\
Unión & 123,055 & 122,075 & 121,901 & 121,537 \\
Europea & & & & \\
Estados & 76,023 & 75,068 & 77,139 & 77,252 \\
Unidos & & & & \\
México & 9,311 & 9,472 & 9,658 & 9,784 \\
Mundo & 491,283 & 497,988 & 509,636 & 518,803 \\
\hline
\end{tabular}

Fuente: http://www.fao.org 
La relevante participación de Nueva Zelanda en el comercio mundial de lácteos se basa en que sus amplios excedentes de producción respecto a su pequeño mercado interno, son altamente competitivos. Así, este país exporta en lácteos el equivalente a 11 mil millones de litros de leche, lo que representa 80 por ciento de su producción.

\section{Principales mercados de exportación}

De acuerdo con información del Ministerio de Agricultura y Silvicultura del gobierno de Nueva Zelanda, en 2004 el principal mercado de exportación (44 por ciento) fue la región de Asia, destacando los mercados de China, Filipinas, Japón y Malasia; en importancia le siguieron la Unión Europea con 13 por ciento, Estados Unidos 12 por ciento, el Medio Oriente 12 por ciento y México 6 por ciento. La participación que tiene Asia como principal destino de las exportaciones lácteas de Nueva Zelanda ha sido propiciada por una serie de factores como el creciente nivel de ingresos de la población en la región, y los cambios en los patrones de consumo, así como por la diversificación de mercados que emprendió el gobierno de Nueva Zelanda a partir de 1972, año en que el Reino Unido, principal mercado de exportación, decidiera formar parte de la hoy conocida Unión Europea. A finales de los sesenta, a Nueva Zelanda no le quedó otro camino que buscar nuevos mercados, ya que sus exportaciones de lácteos hacia el Reino Unido concentraban 90 por ciento de su producción. ${ }^{7}$

Entre los principales productos lácteos de exportación, la leche entera en polvo continúa siendo importante, ya que representa más de 30 por ciento del valor total de las exportaciones; le siguen la mantequilla con 18 por ciento, queso 17 por ciento y finalmente leche descremada en polvo y caseína, ambas cada una con 14 por ciento del valor total de exportación en 2004. En su conjunto, estos cinco productos representaron 95 por ciento del valor total de exportaciones en 2004.

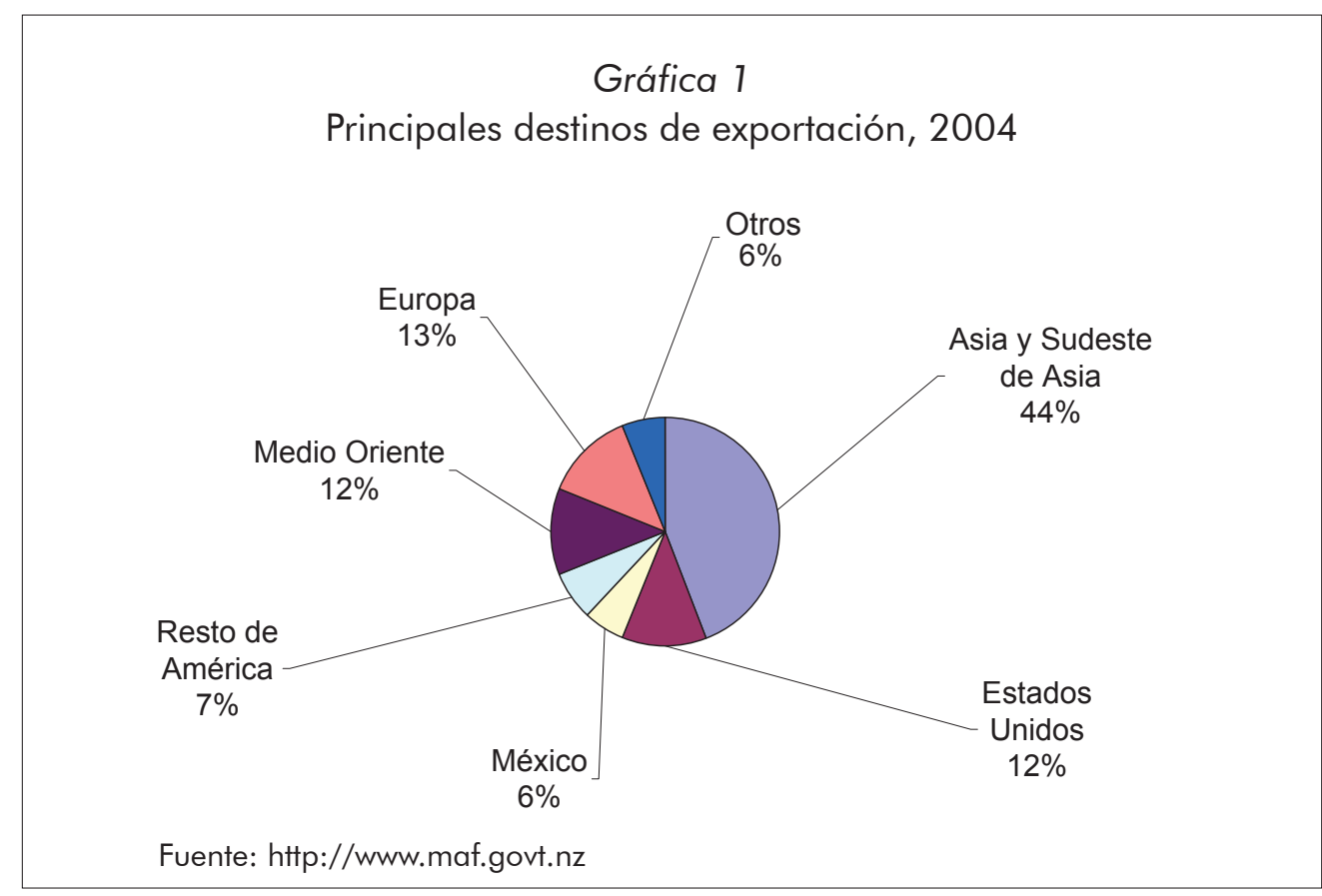

MÉXICO YLACUENCADEL PACÍFICO vol. 8, núm. 24 / enero - abril de 2005 


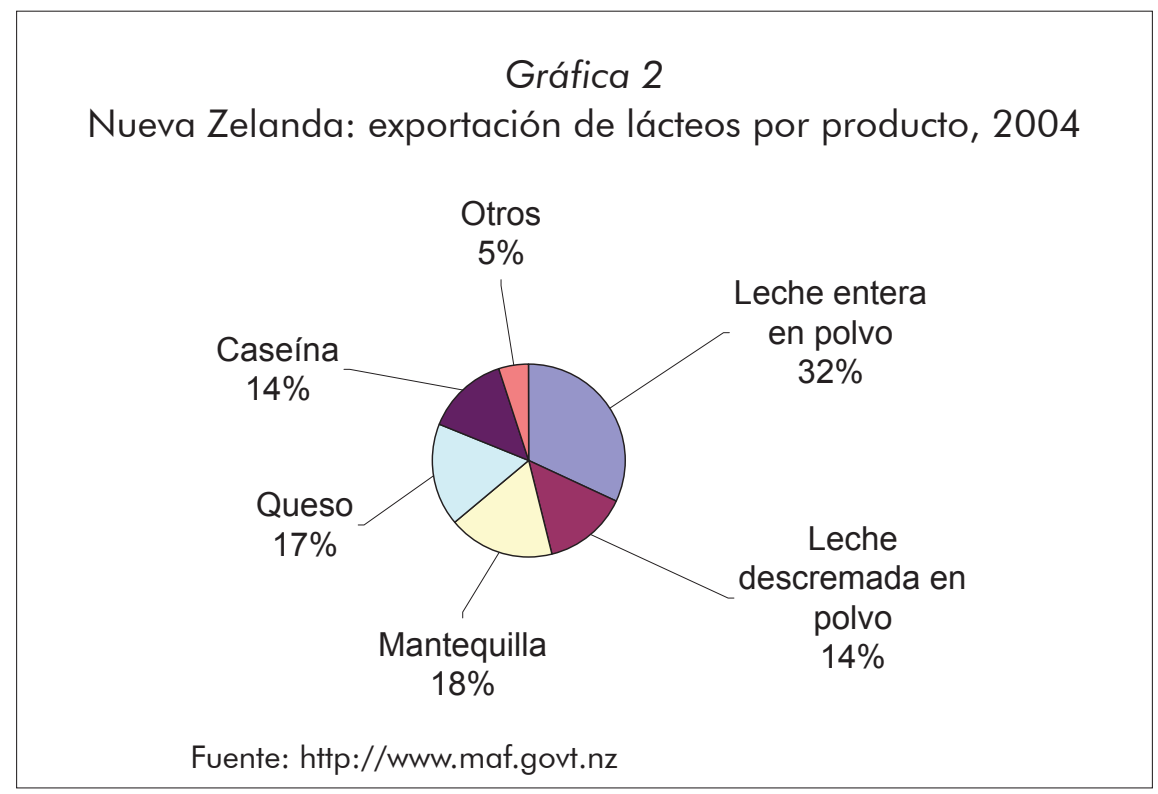

\section{Cambios estructurales en la industria lechera}

Después de la Segunda Guerra Mundial, Nueva Zelanda adoptó una fuerte política proteccionista, la cual se intensificó a finales de los setenta y principios de los ochenta en un intento por conservar la prosperidad de los años cincuenta. ${ }^{8}$ Sin embargo, a partir de 1984, al igual que en muchos otros países en la década de los ochenta, esta nación inició un programa importante de liberalización económica. Los cambios en la economía de Nueva Zelanda durante los ochenta se caracterizaron por su amplitud, la consistencia de las reformas y el bajo nivel de liberalización por el que comenzaron. Muchos programas de la agricultura cambiaron dramáticamente: los esquemas de apoyo al precio fueron eliminados, se terminaron los préstamos para el desarrollo de las granjas y el subsidio a fertilizantes; además, la función del Banco Rural fue reorientada poco a poco hacia la comercialización de sus actividades, lo que provocó el incremento en las tasas de interés que, junto con la caída del ingreso agrícola, condujo a una crisis de endeudamiento rural. ${ }^{9}$

La liberalización en la economía de Nueva Zelanda implicó cambios en el sector agropecuario en general y en el sector lácteo en particular. Estos cambios propiciaron una mayor concentración de la producción de leche; por ejemplo, a mediados de los ochenta el número de compañías lecheras (principalmente cooperativas) que existían en Nueva Zelanda eran 30, mientras que para 1998 quedaban solamente ocho. Este proceso condujo finalmente a una de las fusiones más grandes en 2001, en la que las dos principales cooperativas lecheras de Nueva Zelanda (New Zealand Dairy Group y Kiwi Dairies) y la Compañía Lechera de Nueva Zelanda, (The New Zealand Dairy Board, NZDB), acordaron formar una cooperativa conocida formalmente como Compañía Lechera Global (Global Dairy Company), ahora conocida como Grupo Cooperativo Fonterra (Fonterra Cooperative Group). Actualmente Fonterra es la cooperativa más grande de 
Nueva Zelanda, ya que recibe y procesa alrededor de 95 por ciento de la leche producida en el país.

El antecedente más lejano de Fonterra inició en 1923 con la creación del Consejo para el Control de la Exportación de Productos Lácteos (The Dairy Produce Export Control Board), el cual fue creado por iniciativa del gobierno con el fin de controlar todas las exportaciones de lácteos bajo el argumento de que las compañías procesadoras eran demasiado pequeñas para que por su propia cuenta pudieran exportar con eficiencia. Posteriormente este consejo sería conocido como The New Zealand Dairy Board (NZDB), el cual en 1961 adquirió legalmente el estatus de exportador único. Dicho estatus creaba serios problemas de libre competencia debido a que, como el mercado de Nueva Zelanda era y continúa siendo demasiado pequeño, cualquiera que se iniciara en este negocio tendría que exportar para ampliarse; sin embargo, para hacerlo se debía solicitar autorización a los competidores propietarios de la compañía exportadora, lo cual implicaba una clara desventaja en términos de competencia. Entre otros factores, éste era uno de los puntos más cuestionados de la política gubernamental, por lo que finalmente se logró impulsar un cambio estructural con el propósito de eliminar esta condición de exportador único. ${ }^{10}$ Con la Ley de Reestructuración de la Industria de la Leche en 2001, el estatus de exportador único que mantenía la NzDB quedó eliminado; asimismo, se autorizó la fusión entre la NZDB y las dos cooperativas más grandes del país para dar origen a Fonterra; con ello, la responsabilidad de comercializar los lácteos en el mercado internacional, que otrora tenía la NZDB, pasa, al menos de manera temporal, a ser una responsabilidad de Fonterra.
Con la reestructuración y la creación de Fonterra se puso fin al monopolio de exportador exclusivo de productos lácteos con esta ley, aunque conserva su función de principal exportador debido a su experiencia y al volumen de leche que acopia y procesa; sin embargo, legalmente perdió su condición de monopolio de exportador; asimismo, de acuerdo con la ley la empresa mantendrá por el momento licencias exclusivas de exportación a determinados mercados restringidos, ${ }^{11}$ que estarán vigentes en algunos casos hasta 2007, y en otros hasta $2010 .^{12}$

Finalmente, según datos de la propia empresa Fonterra es actualmente la compañía más grande de Nueva Zelanda; es propiedad, bajo la modalidad de cooperativa de más de 12 mil ganaderos; sus exportaciones representan más de 20 por ciento del total realizadas por el país y participa con 7 por ciento del producto interno bruto, PIB nacional. Es además la empresa privada de Nueva Zelanda que más invierte en investigación y desarrollo, con un gasto anual aproximado de 95 millones de dólares neozelandeses, además de tres millones adicionales que recibe del gobierno vía la Fundación para la Investigación, la Ciencia y la Tecnología (Foundation for Research, Science and Technology). ${ }^{13}$

\section{Los subsidios al sector lácteo}

Los subsidios agrícolas, que son otorgados principalmente por países desarrollados, provocan incrementos en la producción que finalmente se traducen en una baja en los precios a escala internacional; esto hace más difícil que los países pobres puedan competir con sus productos primarios en un mercado altamente subsidiado. Aún más, debido a la baja en los precios internacionales de algunos productos 
primarios, muchos países pobres optan por incrementar la importación de estos productos, lo cual provoca serios daños a su sector agropecuario interno. Para el caso de Nueva Zelanda, que es un país desarrollado pero muy productivo en la elaboración de leche con fuerte presencia en los mercados internacionales, la baja en los precios de la leche provocados por los elevados subsidios que se destinan a la producción en otros países tienen una repercusión directa en el sector lácteo de Nueva Zelanda, en tanto que se reducen los niveles de ingreso del sector.

Entre los países desarrollados, Nueva Zelanda es el que otorga menos subsidios a su sector lácteo. Una forma de medir estos apoyos es mediante un indicador conocido como la estimación de la ayuda al productor, la $\mathrm{PSE}^{14}$ (producer support estimate, en inglés), antes conocido como el equivalente del subsidio al productor; este indicador es una medida adoptada por la Organización para la Cooperación y el Desarrollo Económico (OCDE) para medir el grado de ayuda a la agricultura. En el caso de Nueva Zelanda, la PSE otorgada al productor de leche entre 2001 a 2003 se ha mantenido en 1 por ciento anual, que contrasta con 9 por ciento en el periodo 1986-1988; sin embargo, en ese entonces era muy bajo respecto a las estimaciones de ayuda al productor que realizaban las demás economías desarrolladas. En 2003 la diferencia en la ayuda otorgada por los gobiernos al sector lácteo continúa siendo amplia. En Estados Unidos fue de 45 por ciento, en Japón 77 por ciento, Canadá 59 por ciento, Unión Europea 51 por ciento, México 33 por ciento, Corea del Sur 68 por ciento y finalmente Australia con 15 por ciento. ${ }^{15}$

Otro indicador que utiliza la OCDE para evaluar la ayuda otorgada a productores agropecuarios se conoce como el coeficiente de protección nominal (NPC, por sus siglas en inglés), que es un indicador muy simple utilizado para evaluar el nivel de protección resultante de distorsiones de mercado. Con éste se mide la relación entre el precio interno que reciben los productores por su producto y el precio en el mercado internacional de ese producto. En México, por ejemplo, para el caso de la leche, este indicador fue de 1.46 en 2003; esto reflejaba que en ese año el precio promedio que recibieron los productores de leche en México fue 3.22 pesos por litro; sin embargo, en el mercado internacional el precio en la frontera era de 2.20 pesos el litro, lo que significaba que había una ayuda implícita a los productores de leche en México de 46 por ciento. En Estados Unidos el indicador fue 1.76, en Corea del Sur 3, en la Unión Europea 1.98, en Canadá 2.31, en Japón 4.19 y en Nueva Zelanda y Australia 1, lo cual indica que en estos países no hubo ayuda implícita, o que el precio que recibieron los productores de leche fue igual al internacional.

Es importante aclarar que este indicador, el NPC, forma parte de lo que se conoce como el apoyo del precio de mercado (market price support, MPs) y es uno de los componentes que tiene una proporción de aproximadamente dos tercios dentro de la PSE, es decir, más de 60 por ciento del valor de la PSE se calcula con la diferencia entre los precios internos de los productos agropecuarios y los precios internacionales. ${ }^{16}$ Sin embargo, si se considera que son precisamente los apoyos que reciben los productores agrícolas en países desarrollados los que provocan incrementos en la producción y la oferta mundial, que finalmente se traducen en una caída en los precios internacionales, resulta cuestionable el tamaño de la ayuda implícita que 
resalta en los países desarrollados con este indicador. De esta forma, probablemente si no hubiera subsidios tan elevados la producción mundial se reduciría, el precio aumentaría y la diferencia entre los precios en frontera y los internos serían quizás menores, con lo que tanto la PSE como el NPC para el caso de México y otros países, serían menores.

\section{Conclusión}

Debido a que Nueva Zelanda utiliza un sistema de producción de leche basado en el pastoreo con bajo uso de granos, los rendimientos por bovino son inferiores hasta en 50 por ciento de la producción de leche, comparados con los sistemas estabulados y de un uso mayor de granos concentrados; sin embargo, es ésta también la razón de que los costos de producción sean de los más bajos dentro de los países desarrollados. Al mismo tiempo, ya que el sector lácteo es uno de los más regulados, principalmente en los países desarrollados, esto provoca que los precios en el mercado internacional se encuentren deprimidos, lo que a su vez influye, por una parte, en que los potenciales ingresos adicionales de la industria de leche de Nueva Zelanda se vean reducidos y, por otra, en países pobres como México (donde se importa alrededor de 20 por ciento de su consumo interno) se tiende a desalentar la producción y a incrementar las importaciones. me

\section{Notas}

1 http://www.agritech.org.nz/dairy.shtml, p. 2.

2 Fideicomisos Instituidos en Relación con la Agricultura, FIRA, (2001) Boletín informativo, núm. 317, vol. xxxIII, p. 38. http://www.siap. sagarpa.gob.mx

3 Ibídem, p. 40.

4 Meter, Martin, y Phantipa Puangsumalee (s/f) Farm performance in the Australian and New Zealand dairy industries. Australia: Australian Bureau of Agricultural and Resource Economics. www.abareconomics.com

5 Velázquez, Victor Manuel, Roberto García M., y Miguel Martínez D. Miguel (1998) "Análisis comparativo de los costos de producción de leche de vaca en siete países", Políticas Agrícolas, vol. III, núm. 3.

6 Se toman en cuenta los 15 países que formaban la Unión Europea hasta 2003 (Austria, Bélgica, Dinamarca, Finlandia, Francia, Alemania, Grecia, Irlanda, Italia, Luxemburgo, Holanda, Portugal, España, Inglaterra y Suecia). A partir de 2004 se adhirieron 10 más.

7 http://europa.eu.int/

8 Evans, Lewis (2004) Structural reform: the dairy industry in New Zealand, APEC high level conference on structural reform, 8-9 de septiembre, Tokio, p. 4.

9 www.ifama.org/ (Changes in risk perception and risk management strategies in New Zealand dairy farming), p. 4.

10 Evans, op. cit., p. 8.

11 http://www.wto.org/english/tratop_e/tpr_e/s1154_e.doc

12 Fonterra se beneficiará de licencias exclusivas de exportación hasta el 30 de junio de 2007 para las exportaciones al mercado de la República Dominicana, hasta el 31 de julio de 2007 para las exportaciones a determinados mercados de Canadá, hasta el 31 de diciembre de 2007 para las exportaciones a determinados mercados de la Comunidad Europea, hasta el 31 de diciembre de 2008 y 31 de diciembre de 2009 para las exportaciones de determinados quesos al mercado de Estados Unidos y, finalmente, tendrá licencia exclusiva hasta el 31 de marzo de 2010 para las exportaciones al mercado japonés.

13 http:www.fonterra.com

14 La estimación de ayuda al productor es un indicador del valor de las transferencias monetarias a la agricultura que resultan de las políticas agrícolas en un año dado.

15 OCDE (2004) Agricultural policies in OECD countries at a glance.

16 http://www.oecd.org/dataoecd/63/8/32035391. pdf, p. 4. 
La serie "Cuenca del Pacifico: retos y oportunidades para México" es una colección integrada por cinco volúmenes, bajo la coordinación general de Melba E. Falck Reyes.

Volumen 4. What's in a Name? Globalization, Regionalization, and APEC

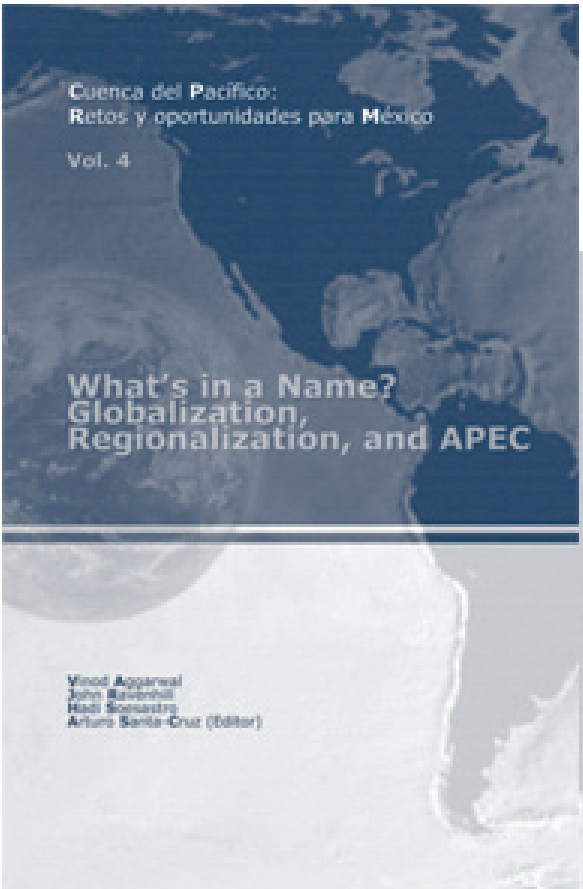

Volumen 1. Economia y cultura en la Cuenca del Pacifico.

editado por Geneviève Marchini.

Volumen 2. El modelo de desarrollo asiático. Relevancia para México, editado por Melba E. Falck Reyes y Roberto Hernández Hernández.

Volumen 3. Jalisco y su inserción en la Cuenca del Pacifico, editado por Dagoberto Amparo y Melba E. Falck Reyes.

Volumen 4. What's in a Name? Globalization, Regionalization, and APEC,

editado por Arturo Santa Cruz.

Volumen 5. Agricultura en la Cuenca del Pacifico,

editado por Roberto Hemández Hernández.
Vinod Aggarwal

John Ravenhill

Hadi Soesastro

Arturo Santa-Cruz (editor)

Primera edición, 2003

Universidad de Guadalajara

Precio \$ 130.00

This volume presents a critical evaluation of the processes of globalization and regionalization as instantiated in the Asia Pacific Economic Cooperation mechanism (APEC). The introductory chapter emphasizes the aspirational nature of the Pacific Rim as a region, and argues that its possible materialization would, paradoxically, be a sign of deeper globalization. The second chapter looks at APEC as process, emphasizing its peculiar characteristics and ultimate goal: community building. The third chapter undertakes a critical evaluation of APEC. Focusing on the three pillars of the organization (trade liberalization, trade facilitation, and economic and technical cooperation), it shows the rather meager progress achieved so far. It then poses the questions of whether APEC has been overburdened with expectations and whether its institutional design is adequate. Finally, Chapter Four makes an explicit connection between a "transregional" organization like APEC and the global process of trade liberalization through the World Trade Organization (WTO). This chapter presents an analytical fromework on modes of trade liberalization in light of APEC's role in trade liberalization since the Seattle 1999 WTO Summit. Again, the outcome is not heartening for advocates of free trade. Thus, the global stage seems set for the flowering of regional and bilateral integration whereas the Pacific Rim, as a region inscribed in a globalized economy, seems to remain a distant aspiration.

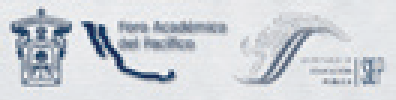

De venta en: libreria el Kiosco, Centro Universitario de Ciencias Sociales y Humanidades,

Av. Alcalde esquina Av. de los Maestros, edificio G, planta baja, Guadalajara, Jalisco 